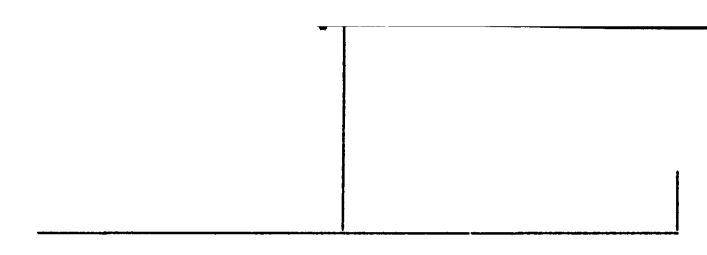

Rev. Latinoam. Psicopat. Fund., VII, 4, 155-164

\title{
Estudos psicopatológicos na psiquiatria atual
}

\author{
Adriano Carvalho Tupinambá Rodrigues \\ Cláudio E.M. Banzato
}

\section{Introdução}

Desde o lançamento de Allgemeine Psychopathologie, de Karl Jaspers, em 1913, em que bases metodológicas para o desenvolvimento da psicopatologia geral foram propostas de modo sistemático, a investigação psicopatológica tem apresentado diferentes contornos. É preciso salientar que a importância central de Jaspers decorre não apenas da identificação das condições necessárias para a consolidação dessa disciplina, incluindo aí a descrição das sucessivas "instâncias heurísticas" que se deve percorrer para o exame prolífico dos fenômenos psicopatológicos, mas também de sua clareza sobre o hiato epistêmico existente entre os métodos empíricos e teóricos de tratamento dos fenômenos mórbidos, e sobre a indispensável articulação entre fatos e teorias. Assim, além de superar inúmeros "preconceitos" reducionistas, o desafio posto para qualquer projeto psicopatológico seria evitar a um só tempo a esterilidade de um empirismo ingênuo e o brilho enganador de uma teoria descolada da realidade. Jaspers, sem se comprometer com um instrumental, técnica ou perspectiva particular de enfoque à psicopatologia, procurou, antes, expor as condições gerais a serem satisfeitas por quaisquer projetos psicopatológicos específicos (Jaspers, 2000). 


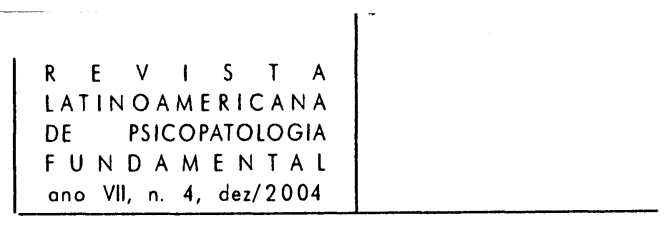

O ideal de projeto investigativo e de edificação de uma psicopatologia geral, em consonância com Jaspers, exigiria não apenas investimentos concentrados numa ou noutra "instância heurística", mas sobretudo uma lúcida articulação das perspectivas envolvidas, entendidas como interdependentes e referenciadas entre si (ibid.). Suspeitamos, porém, que ainda hoje, em meio à diversidade de abordagens psicopatológicas, prevalecem os estudos que tratam exclusivamente de determinados aspectos isolados, em detrimento dos demais. Se esse é realmente o caso, o programa investigativo delineado por Jaspers estaria ainda em seus primórdios e, a julgar por um editorial do American Journal of Psychiatry, de 1998, mesmo tais iniciativas preliminares parecem estar perdendo terreno na psiquiatria atual. No referido editorial, contemplando o panorama psiquiátrico do momento, a norte-americana Nancy Andreasen formula a questão com muita precisão e contundência:

... where is the good old-fashioned clinical research? Where are studies that examine epidemiology, descriptive psychopathology, and course and outcome? Studies in this area are becoming increasingly rare, since they are no longer perceived as "sexy," "cutting edge," "sophisticated," or even "scientific". They are incredibly low tech. They only require having a thinking brain/mind, observing many patients, and recording observations and measurements in a systematic way that can be analyzed by using statistical methods that range from very simple to very complicated. In the United States an older generation of clinical researchers who led the field for many years have died (...) or are dying out. Very few younger investigators are emerging to replace them. The word is out - if you want to succeed as a serious scientist, you need to do something relatively basic. Fortunately, the Europeans still have a proud tradition of clinical research and descriptive psychopathology. Someday in the twenty-first century, after the human genome and the human brain have been mapped, someone may need to organize a reverse Marshall plan so that the Europeans can save American science by helping us figure out who really has schizophrenia or what schizophrenia really is. The fledging American school of descriptive psychopathology will have become extinct. Yet we cannot apply the potentially great fruits of the Human Genome Project to complex mental illnesses if we no longer have clinical investigators who have devoted their research careers to conceptualizing the nature and definitions of symptoms, syndromes, diseases, or diagnoses. (1998)

O presente artigo se propõe a identificar alguns dos tipos mais comuns de investigação psicopatológica atualmente levados a cabo no campo da psiquiatria. Para tanto, empreendemos uma revisão não sistemática da literatura científica, com ênfase no ano de 2004, utilizando as bases de dados Medline, PsycInfo e SCI. Selecionamos determinados trabalhos que consideramos ilustrativos e 


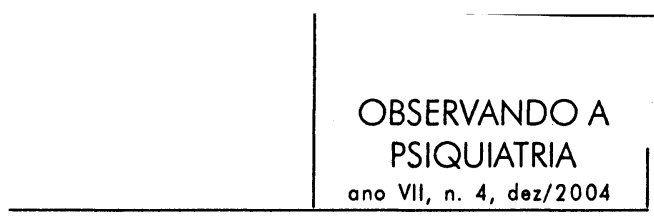

procuramos apontar algumas das principais virtudes e limitações destes tipos de estudos, suas conquistas, suas ambições explicativas, além dos aspectos freqüentemente negligenciados pelos mesmos.

\section{Tipos de investigação psicopatológica em psiquiatria}

Embora nem sempre seja tarefa fácil identificar os objetivos específicos dos estudos, posto que não raramente nos deparamos com uma conflução inadvertida de propósitos - cuja ambição implícita seria abarcar todas as etapas sucessivas necessárias à demonstração da hipótese em questão - podemos, aproximadamente, separar os estudos em dois grupos mais importantes, segundo seus fins e resultados mais significativos.

Um primeiro tipo de estudo psicopatológico, de longa tradição na psiquiatria, é o chamado fenomenológico-descritivo. Entre estes se contam mesmo alguns de desenho mais simples, que se propõem ao mero registro de manifestações isoladas ou quadros sintomáticos em populações específicas, assim como os que pretendem realizar descrições mais detalhadas de tais quadros, apurar suas prevalências e incidências, ou estabelecer distinções fenomenológicas entre populações. Trabalhos como o de Chang et al. (2004), em que o aspecto central é a descrição da síndrome de Tourette numa população bastante específica, como a de "adolescentes orientais", são representativos desse subgrupo de estudos. Freqüentemente, a análise e discussão dos resultados desse tipo de trabalho os comparam a resultados obtidos por estudos junto a populações diferentes (neste caso, estudos com pacientes de países ocidentais). Contudo, enquanto nesses trabalhos o objetivo principal é a "descrição", sendo a "comparação" permitida um desdobramento, estudos como o de Turkel et al. (2004) e o de Winkler et al. (2004) são mais especificamente dirigidos a este segundo propósito e já incluem duas populações distintas em suas avaliações. Nestes dois casos, especificamente, a atenção se dirige a possíveis diferenças sintomáticas do transtorno obsessivo compulsivo e quadros depressivos, respectivamente, em populações feminina e masculina. Além disso, na medida em que comportamentos de determinadas populações tentam ser compreendidos em função da ocorrência de certos sintomas ou déficits em seus membros, e com seus testes de hipótese baseando-se em diferenciais de freqüência, os estudos em que o aspecto descritivo apresenta características epidemiológicas continuam tendo espaço. Exemplo disso é a determinação da prevalência de sintomas psicóticos em adolescentes holandeses detentos, por Vreugdenhil et al. (2004). Outros autores, nesta mesma linha geral, buscam enfatizar aspectos supostamente negligenciados 


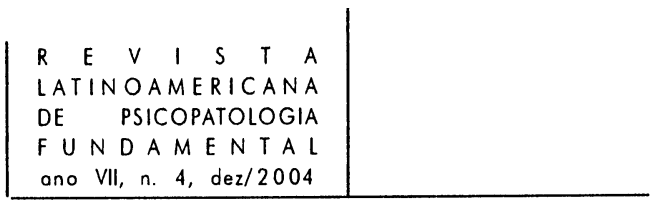

pela maioria dos demais estudos, ou questionar formulações consagradas pelas descrições "clássicas" dos fenômenos. Sanjuan et al. (2004), por exemplo, ressaltando o notável volume de estudos disponíveis sobre alucinações visuais em seu aspecto desprazível e indesejável pelo paciente, destacam as "alucinações auditivas de conteúdo agradável", buscando aferir sua prevalência e relacionandoas a outras manifestações e prognóstico. Caso semelhante é o do trabalho de Pasquini et al. (2004) em que, comentando sobre os critérios diagnósticos para depressão unipolar nas atuais classificações de transtornos mentais, examina a relevância de manifestações não criteriais - como hostilidade, irritabilidade e agressividade - em pacientes com tal diagnóstico. Investigações desta mesma linha, como a realizada por Appelbaum et al. (2004), em que se testa a convicção com que delírios são sustentados e sua resistência a transformações ao longo do tempo, lançam dúvidas até mesmo sobre descrições clínicas referendadas pela tradição. Tais abordagens, oferecendo novas perspectivas dos fenômenos estudados, permitem o registro de correlações antes insuspeitas ou impossíveis.

Entre as investigações fenomenológico-descritivas com maior poder de gerar novas hipóteses, temos aquelas que se valem de técnicas estatísticas mais sofisticadas, tais como as análises fatoriais, que possibilitam a discriminação de dimensões, clusters ou subgrupos de sintomas no interior de uma condição até então tratada como única do ponto de vista fenomenológico. Enquanto estudos como o de Maggini e Raballo (2004) - em que uma estrutura tetra-fatorial foi achada para a alexitimia - e o de Denys et al. (2004) - em que seis clusters sintomáticos foram encontrados para o transtorno obsessivo-compulsivo - põem em xeque a unidade nosológica desses quadros, outros, como o de Richardson et al. (2004), utilizam esse recurso para demonstrar como determinados comportamentos (como violência sexual) não guardam correlação com um único padrão psicológico, mas diversos. Assim, além de poder servir a propósito semelhante ao do tipo de estudo anteriormente mencionado, ou seja, à geração de novos recortes fenomenológicos, é também um recurso que tem o mérito de oferecer uma visão do padrão de aglutinação de variáveis - neste caso, sintomas ou manifestações afins - e, com isso, criar as bases para projetos de validação categorial, com possíveis implicações nosográficas (Lahey et al., 2004). Como limitação comum aos estudos deste grupo temos a falta de apresentação e defesa de seus pressupostos conceituais e metodológicos e a insuficiente exploração das consequiências teóricas de seus achados.

Uma segunda categoria abrangente de estudos trata das correlações entre fenômenos psicopatológicos e eventos de diversas ordens. Assim, encontramos um primeiro subgrupo de estudos em que as modalidades de associação estabelecidas pressupõem que o objeto central da investigação, o fenômeno psicopatológico, possa ser sistematicamente relacionado a uma dada variável 


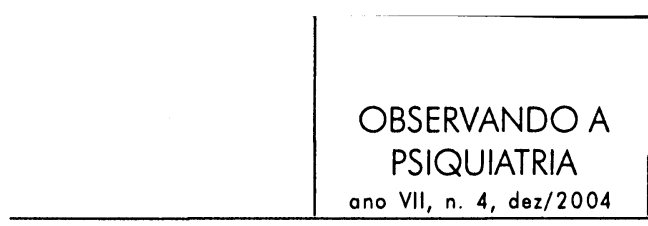

hipotética. Busca-se estabelecer, acerca de sintomas isolados ou constelações de sintomas (síndromes), seus determinantes causais e/ou a influência sobre os mesmos de fatos vitais (Pert et al., 2004; Lang et al., 2004; Faravelli et al., 2004), modo de início do processo (Tulio-Henriksson et al., 2004), modo de evolução (Benazzi, 2004), disposições neurofisiológicas (Chau et al., 2004; Halari et al., 2004) ou funções neuropsicológicas (Bozikas et al., 2004a; Green et al., 2004; Koren et al., 2004; Keshavan et al., 2004; Bozikas et al., 2004b). Representantes deste subgrupo de estudos são aqueles em que se buscam associações entre perda parental precoce e o desenvolvimento ulterior de transtornos de personalidade, entre maus-tratos na infância e sintomas psiquiátricos/re-exposição na idade adulta, ou entre vitimização por violência sexual e posterior psicopatologia. No patamar biológico, estudos deste tipo são representados por aqueles que averiguam associações entre circuitos neurais e síndromes psiquiátricas, assim como entre perfis endócrinos e a intensidade de determinado sintoma (como a relação entre hormônios sexuais e o grau de disfunção cognitiva em pacientes esquizofrênicos). Um volume considerável de estudos, atualmente, também busca correlações entre determinados parâmetros neuropsicológicos e quadros psiquiátricos ou manifestações específicas; por exemplo, entre funcionamento cognitivo e o quadro psicopatológico global na esquizofrenia, ou entre funcionamento "meta-cognitivo" e o grau de insight em quadros psicóticos. No sentido inverso, encontram-se trabalhos em que se espera das associações postuladas alguma capacidade prognóstica, isto é, de predição de consequiências como evolução clínica (Biederman et al., 2004; Tulio-Henriksson et al., 2004), resposta terapêutica, comorbidades (Biederman et al., 2004), qualidade de vida (Hofer et al., 2004), comportamentos manifestos (Buckley et al., 2004) e mesmo surgimento de outros quadros sintomáticos a partir do fenômeno estudado. Existem ainda estudos que, combinando os dois tipos de abordagem acima (busca de "antecedentes causais" ou "resultantes" das manifestações psicopatológicas), utilizam os fenômenos psicopatológicos como variáveis mediadoras (Kim et al., 2004; Marmorstein et al., 2004).

Parte desses estudos abordando aspectos neurofisiológicos, neuropsicológicos, cognitivos e bioquímicos são compatíveis com um projeto que vem se desenhando recentemente, a busca pelo estabelecimento dos chamados "endofenótipos" [que podem ser definidos como aqueles componentes mensuráveis, intermediários entre a doença e o genótipo distal (Gottesman e Gould, 2003). A complexa determinação poligênica dos principais quadros psiquiátricos fez com que alguns pesquisadores passassem a considerar mais factível a busca alternativa do substrato genético de certas manifestações ou disposições psicopatológicas presumivelmente mais simples. Em última instância, pretende-se com isso viabilizar a organização de um modelo diagnóstico e classificatório com melhor 


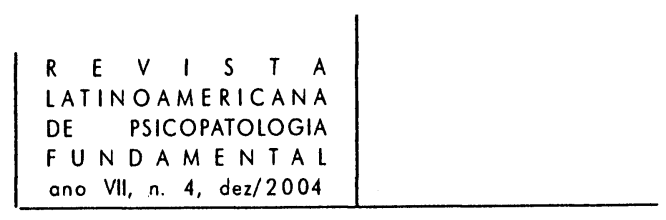

correspondência com as bases neurobiológicas dos fenômenos psicopatológicos (ibid.).

\section{A aspiração jasperiana e o que já foi alcançado}

Pelo exposto, fica clara a diversidade das questões colocadas, bem como dos tipos de estudos psicopatológicos que tentam respondê-las. Praticamente todas as perspectivas a partir das quais se estudam os fenômenos psíquicos, em suas interfaces com a psicopatologia, ajudam a compor o complexo cenário das abordagens atualmente praticadas. Aí estão incluídos estudos clínicos, epidemiológicos, forenses, neuropsicológicos, neurobiológicos, entre outros. Entretanto, em que pese a maior minúcia dos dados que se perquire, o aprimoramento do instrumental técnico disponível e a sofisticação dos desenhos dos estudos, que certamente representam um avanço das investigações na área, ainda assim parece-nos discutível a idéia de que houve uma evolução metodológica. A despeito da multiplicidade de métodos e objetivos aqui inventariados, o enquadramento numa perspectiva científica naturalista parece ser a regra, sem no entanto ir além de sua etapa observacional. Não se trata de criticar o projeto científico naturalista, mas sim as limitações de seus resultados quando tomados isoladamente, sem a devida articulação teórica.

Um regresso a Jaspers, de fato, nos revela que àquela oportunidade o autor já sugeria que, não apenas a atitude realmente científica exigia que o método se aplicasse a fatos, como também manifestava seu incômodo com a constatação de que na bibliografia psiquiátrica da época predominavam as teorias não legitimadas pela observação:

Encontram-se muitos discursos sobre possibilidade, muita coisa abstrata, muitas construções feitas de imaginação sem o conteúdo de uma experiência verdadeira. Por isso, no estudo dos trabalhos publicados como na própria investigação, temos sempre de perguntar: qual o acervo dos fatos? (...) Face a pensamentos pobres de experiência deve-se perguntar se não são de se recusar por serem vazios (...) o mais possível, não se deve perder tempo procurando esclarecer querelas sobre pensamentos e projetos sem seriedade. $(2000$, p. 36)

Além disso, esse autor já apontava as casuísticas, a estatística, os experimentos, as investigações sobre hereditariedade e medidas de correlações caracterológicas - métodos que continuam sendo utilizados até hoje - como alguns dos recursos técnicos a serem empregados com este fim.

Todavia, Jaspers também propunha claramente que, se por um lado, a fundamentação da investigação se dá pelo recurso aos fatos e por meio do 


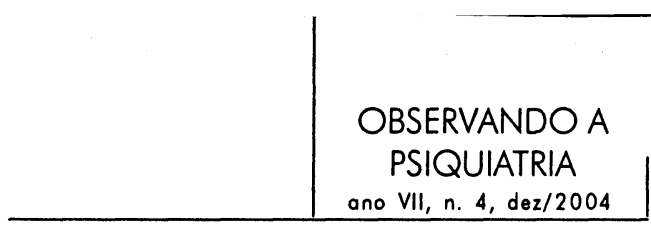

apreensível, por outro, sua finalidade nestes não se encerra, demandando sempre uma integração compreensível dos dados adquiridos, que, além das correlações imediatas entre os objetos em questão, permita também a apreciação do psiquismo e suas manifestações patológicas como uma totalidade.

O trabalho científico só fará progressos se analisar, referir uns aos outros fenômenos particulares. No entanto, se ficar nisso só, morrerá, não podendo distinguir o essencial do não essencial. Pois, então, cairá no comodismo de enumerar apenas fenômenos esparsos. Ao invés, deve sempre ser movido por idéias de totalidade sem sucumbir, porém, à tentação de querer apreender diretamente as totalidades por meio de antecipações fáceis. Nestas o psicopatologista se embriaga com frases e se restringe por meio de um falso domínio do todo, de uma pretensa percepção de forças psíquicas englobantes. Nosso trabalho de investigação deve, por fim, conservar, como último horizonte, a consciência da amplidão do ser humano. Todo que se puder investigar empiricamente no homem, é sempre parte, aspecto, é sempre relativo, mesmo que seja a totalidade empiricamente mais compreensiva. (2000, p. 45)

Acreditamos que esta aspiração jasperiana a uma psicopatologia que evoluísse para além das correlações imediatas e empiricamente respaldadas até um entendimento global das conexões psicopatológicas, tem sido freqüentemente negligenciada nas principais linhas investigativas em psicopatologia na psiquiatria contemporânea. Enquanto as próprias codificações nosográficas são entendidas como organizações "ateóricas" de constelações de sintomas, não é de se estranhar que os testes de hipóteses nos estudos psicopatológicos também visem tãosomente a confirmação estatística de fatos clinicamente observados. Em que pese a relevância do elemento empírico para a construção do conhecimento e a importância das informações reunidas na literatura sobre os fenômenos mentais e seus correlatos, sejam comportamentais, epidemiológicos, prognósticos ou neurobiológicos, o investimento em tal instância não parece ser seguido, via de regra, por tentativas de promover uma organização compreensiva e significativa das inúmeras correlações estabelecidas entre fenômenos particulares.

Podemos questionar, assim, a utilidade de se prosseguir com os estudos de correlação, a menos que se procure extrair conseqüências teóricas da imensidão de dados ora disponíveis. Por vezes, o maior detalhamento ou minúcia alcançada na formulação de fenômenos psicopatológicos gera uma falsa impressão de progresso. E, se o horizonte que garantiria um propósito para a continuidade do empenho nos estudos empíricos em psicopatologia - a saber, o emprego dos dados para numa organização dotada de sentido - é atualmente negligenciado, a situação não é menos indeterminada quanto a seu "ponto de partida". Enquanto o mainstream dos estudos em psicopatologia pretende rigor com o exame empírico dos fenômenos, desde os mais básicos, freqüentemente realiza-o de modo 


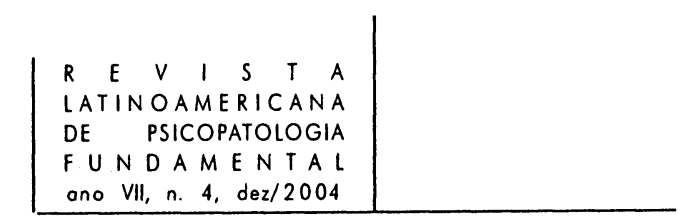

precário, já que o primeiro passo, que é o de delimitação clínica e conceitual do fenômeno não raro deixa a desejar. Alguns psicopatólogos, assim, dão a impressão de que os fenômenos por eles estudados são reconhecidos de forma não problemática por seus pares, enquanto outros, mais críticos, contestam esta possibilidade, sem no entanto se debruçarem sobre a questão.

\section{Conclusão}

Diante do cenário investigativo em psicopatologia aqui apresentado, acreditamos poder afirmar que, da aspiração jasperiana à construção de uma psicopatologia geral teoricamente consistente e empiricamente embasada, obtivemos apenas um complexo mosaico de peças pouco articuladas. Esta disjunção se dá especialmente, segundo entendemos, entre as instâncias teoricamente dependentes (definição dos fenômenos e articulação compreensiva dos dados) e a etapa intermediária de obtenção dos dados a partir dos fatos. Deste modo, a recente preocupação em que se formem novas gerações de psicopatólogos deve também ser seguida pela indagação sobre o quanto estudos "epidemiológicos, de psicopatologia descritiva e sobre curso e prognóstico" (Andreasen, 1998), ainda fazem sentido e nos são suficientes. Como contraponto, sugerimos que é mais em direção a um ideal teórico de síntese de informações a exemplo de outras ciências - que propriamente da formação de novos "observadores", que se favorecerá a psicopatologia. Neste sentido, parece-nos que o futuro da psicopatologia geral, como concebida por Japers, depende da adoção de um pluralismo metodológico e do constante diálogo da tradição empirista em psiquiatria com propostas conceitualmente mais refinadas e teoricamente mais ousadas.

\section{Referências}

ANDREASEN, Nancy. Understanding Schizophrenia: A Silent Spring? American Journal of Psychiatry, Estados Unidos, v. 155, n. 12, p. 1657-59, dez./1998.

Appelbaum, P.S. et al. Persistence and stability of delusions over time. Comprehensive Psychiatry, Nova York, Estados Unidos, v. 45, n.5, p. 317-24, out./2004.

BenazzI, F. Depressive Mixed state: A feature of the natural course of bipolar II (and major depressive) disorder? Psychopathology, Basiléia, Suíça, v. 37, n. 5, p. 207-12, set-out./2004. 


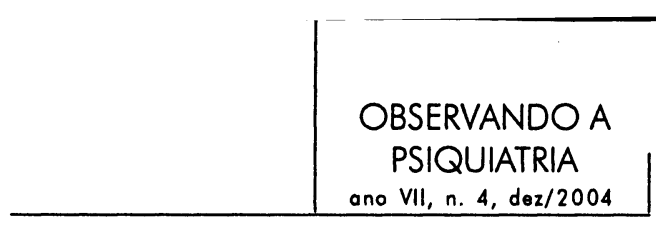

Biederman, J. et al. Phenomenology of childhood psychosis: findings from a large sample of psychiatrically referred youth. Journal of Nervous and Mental Disease, U.S.A., v. 192, n. 9, p. 607-14, set./2004.

BozıKAs, V.P. et al. Relationship between psychopathology and cognitive functioning in schizophrenia. Comprehensive Psychiatry, Nova York, Estados Unidos, v. 45, n. 5, p. 392-400, out./2004a.

Relationship of affect recognition with psychopathology and cognitive performance in schizophrenia. Journal of International Neuropsychological Society, Cambrige, Inglaterra, v. 10, n. 4, p. 549-58, jul./2004b.

BUCKLEY, P.F. et al. Insight and its relationship to violent behavior in patients with schizophrenia. American Journal of Psychiatry, Estados Unidos, v. 161, n. 9, p. 171214, set./2004.

Chang, H.L. et al. Tourette's syndrome: psychopathology in adolescents. Psychiatry and Clinical Neurosciences, Japão, v. 58, n. 4, p. 353-58, ago./2004.

Chau, D.T. et al. The neural circuitry of reward and its relevance to psychiatric disorders. Current Psychiatry Reports, Filadélfia, Estados Unidos, v. 6, n. 5, p. 39199, out./2004.

COOPER, R. What is wrong with the DSM? History of Psychiatry, Reino Unido, v. 15, n. 1, p. 5-25, mar./2004.

DENYS, D. et al. Symptom dimensions in obsessive-compulsive disorder: factor analysis on a clinician-rated scale and a self-report measure. Psychopathology, Basiléia, Suíça, v. 37, n. 4, p. 181-89, jul-ago./2004.

Faravelli, C. et al. Psychopathology after rape. American Journal of Psychiatry, Estados Unidos, v. 161, n. 8, p. 1483-85, ago./2004.

GotTesman, I.I. e GouLd, T.D. The endophenotype concept in psychiatry: Etymology and strategic intentions. American Journal of Psychiatry, Estados Unidos, v. 160, n. 4, p. 636-45, abr./2003.

Green, N.E. et al. Can disorganization of semantic memory account for the abnormalities of thought in schizophrenia - A controiled experimental study. Schizophrenia Research, Amsterdan, Holanda, v. 70, n. 2-3, p. 233-40, out./2004.

Halari, R. et al. The relationship of sex hormones and cortisol with cognitive functioning in schizophrenia. Journal of Psychopharmacology, Bristol, Reino Unido, v. 18, n. 3, p. 366-74, set./2004.

HAYASHI, N. et al. Is there a gender difference in a factorial structure of the positive and negative syndrome scale? A test by structural equation modeling. Psychopathology, Basiléia, Suíca, v. 35, n. 1, p. 28-35, jan-fev./2002.

HoFER, A. et al. Quality of life in schizophrenia: the impact of psychopathology, attitude toward medication, and side effect. Journal of Clinical Psychiatry, Estados Unidos, v. 65 , n. 7 , p. $932-39$, jul./2004. 
JASPERS, Karl. Introdução. In: Psicopatologia Geral. São Paulo: Atheneu, 2000. p. 11-68.

Keshavan, M.S. et al. Correlates of insight first episode psychosis. Schizophrenia Research, Amsterdan, Holanda, v. 70, n. 2-3, p. 187-94, out./2004.

Kıм, C.D. et al. Seasonal differences in psychopathology of male suicide completers. Comprehensive Psychiatry, Nova York, Estados Unidos, v. 45, n. 5, p. 333-39, out./ 2004.

Koren, D. et al. The neuropsychological basis of insight in first-episode schizophrenia: a pilot metacognitive study. Schizophrenia Research, Amsterdan, Holanda, v. 70, n. 2-3, p. 195-202, out./2004.

LAHEY, B.B. et al. The structure of child and adolescent psychopathology: generating new hypotheses. Journal of Abnormal Psychology, Washington, Estados Unidos, v. 113 , n. 3, p. 358-85, ago./2004.

LANG, A.J. et al. Adult Psychopathology and intimate partner violence among survivors of childhood maltreatment. Journal of Interpersonal Violence, Estados Unidos, v. 19, n. 10 , p. 1102-18, out./2004.

MaGgini, C. e Raballo, A. Alexithymia and schizophrenic psychopathology. Acta Biomedica del Ateneo Parmense, Parma, Itália, v. 75, n. 1, p. 40-9, abr./2004.

Marmorstein, N.R. et al. Psychiatric disorders among offspring of depressed mothers: associations with paternal psychopathology. American Journal of Psychiatry, Estados Unidos, v. 161, n. 9, p. 1588-94, set./2004.

PASQUINI, M. et al. Relevance of anger and irritability in outpatients with major depressive disorder. Psychopathology, Basel, Suíça, v. 37, n. 4, p. 155-60, jul-ago./2004.

PERT, L. et al. Parental loss before age of 16 years: A comparative study of patients with personality disorder and patients with schizophrenia in a high secure hospital's population. Psychology and Psychotherapy, Reino Unido, v. 77, n. 3, p. 403-7, set./ 2004.

Richardson, G. et al. Personality-based taxonomy of sexually abusive adolescents derived from the Millon Adolescent Clinical Inventory (MACI). British Journal of Clinical Psychology, Reino Unido, v. 43, n. 3, p. 285-98, set./2004.

Sanjuan, J. et al. Pleasurable auditory hallucinations. Acta Psychiatrica Scandinavica, Copenhagen, Dinamarca, v. 110, n. 4, p. 273-78, out./2004.

TURKEL, R. et al. Gender-related differences among Turkish patients with obsessivecompulsive disorder. Comprehensive Psychiatry, Nova York, Estados Unidos, v. 45, n. 5, p. 362-66, out./2004.

Tulio-Henriksson, A. et al. Age at onset and cognitive function in schizophrenia. British Journal of Psychiatry, Reino Unido, v. 185, n. 3, p. 215-19, set./2004.

VReugdenhil, $C$. et al. Psychotic symptoms among male adolescent detainees in The Netherlands. Schizophrenia Bulletin, Estados Unidos, v. 30, n. 1, p. 73-86, 2004.

WINKLER, D. et al. Gender differences in the psychopathology of depressed inpatients. European Archives of Psychiatry and Clinical Neurosciences, Heidelberg, Alemanha, v. 254 , n. 4, p. 209-14, ago./2004. 